\title{
Effect of the microstructure of mortars with low hydraulicity slag on their behavior in aggressive environments
}

\section{Effet de la microstructure de mortiers à base de laitier de faible hydraulicité sur leur comportement face à des environnements agressifs}

\author{
Hadj sadok Ahmed ${ }^{1}$ \\ ${ }^{1}$ Ecole Nationale Supérieure de l'Hydraulique (ENSH), GEE, Blida, Algérie \\ 1a.hadjsadok@ensh.dz
}

\begin{abstract}
Slag is one of the most used cement additives. Due to its latent hydraulic nature, attached to its hydraulicity, it can contribute to a microstructural modification and an improvement of the durability of the concrete face of aggressive environments. In this study, a low active slag is used in the manufacture of mortars as a substitute for cement, at a maximum rate of 50\%. Firstly, a study of the microstructure with mercury porosimetry was used for determination of microstructural parameters (porosity, diameters and volume distribution). The behavior of mortars in aggressive environments (sodium and magnesium sulphate and seawater) was studied later. Despite the low reactivity of studied slag, its presence especially at $50 \%$ rate, in the long term, has led to a refinement of the microstructure. This effect, among others, led to better resistivity of the mortars in the sulphate environnements.
\end{abstract}

\begin{abstract}
Résumé. Le laitier compte parmi les ajouts cimentaires les plus utilisés. De part son caractère hydraulique latent, lié de son hydraulicité, peut contribuer à une modification de la microstructure et une amélioration de la durabilité du béton face à des milieux agressifs. Dans cette étude, un laitier de faible hydraulicité est utilisé dans la fabrication de mortiers comme substituant au ciment, à un taux maximal de 50\%. En premier temps, une étude de la microstructure à l'aide de la porosimétrie au mercure a été réalisée permettant la détermination des paramètres microstructurales (porosité, diamètres et distribution volumique). Le comportement des mortiers face à des environnements agressifs (sulfates de sodium et de magnésium et eau de mer) a été étudié en suite. Malgré la faible réactivité du laitier étudié, sa présence particulièrement à un taux élevé de $50 \%$, au long terme, a engendré un raffinement de la microstructure. Cet effet, entre autres, a conduit à une meilleure résistivité des mortiers dans les milieux sulfatiques.
\end{abstract}

\section{Introduction}

Le laitier, sous-produit de l'industrie de la fonte, compte parmi les ajouts cimentaires les plus utilisés. Présentant un caractère hydraulique latent, lorsqu'il est présent dans le ciment, son hydratation, liée de son activité hydraulique, conduit à une amélioration de la microstructure (raffinement de la porosité) et à une diminution de quantité de chaux dans la pâte de ciment (réaction pouzzolanique). De ce fait la durabilité du béton contenant du laitier face à des milieux agressifs est généralement améliorée. La dissolution du verre du laitier est assurée par l'attaque hydroxylique (des ions $\mathrm{OH}$ - en présence d'eau) provenant de l'hydrolyse de la Portlandite $\mathrm{Ca}(\mathrm{OH})_{2}$, produit d'hydratation du clinker [1]. Néanmoins, ce processus est conditionné par l'hydraulicité du laitier qui est principalement influencée par sa finesse, sa composition chimique et son contenu en verre [2]. Les produits d'hydratation formés dans la matrice cimentaire sont, principalement, des silicates et des aluminates de calcium hydraté ( $\mathrm{CSH}, \mathrm{CAH})$ additionnelles. La matrice résultante présente une bonne résistance chimique et une structure de pore plus raffiné 
[3]. De ce fait les performances mécaniques et de durabilité du béton son améliorées.

En Algérie, le laitier granulé, produit par le complexe sidérurgique d'El Hadjar, est caractérisé par une moyenne réactivité hydraulique [4]. Une étude antérieure [5] a montré son effet positif sur les propriétés de transport, notamment au long terme, dans des conditions de cure humide. Néanmoins, peu d'études ont été menées sur son effet sur la durabilité dans des milieux agressifs, dépendant essentiellement de la résistance chimiques mais aussi des propriétés microstructurales de la matrice.

\section{Expérimentation}

\subsection{Matériaux et mélanges}

Le laitier de haut fourneau utilisé est un laitier granulé issu du complexe sidérurgique d'El Hadjar. D'une granulométrie $0 / 5 \mathrm{~mm}$, sa structure est vitreuse à plus de $95 \%$. Le laitier a été broyé au laboratoire à une finesse d'environ $4200 \mathrm{~cm}^{2} / \mathrm{g}$ à laide d'un broyeur à boulés. Le ciment utilisé pour la confection des mortiers est un ciment Portland de type CEM I 52,5 N. Les compositions chimiques du ciment et du laitier utilisés sont données dans le tableau 1. Le sable utilisé pour la confection des mortiers est un sable normalisé de granulométrie $0 / 2 \mathrm{~mm}$. Cette étude est réalisée sur des mortiers composés de trois (03) sables et un (01) ciment avec un rapport E/C de 0,5. Trois (03) mortiers M0, M30 et M50 ont été fabriqués en substituant, respectivement, 0,30 et $50 \%$ du poids de ciment par du laitier.

\subsection{Préparation des spécimens et essais réalisés}

Les échantillons de mortier sont coulés dans des moules en acier et compactés en deux couches à l'aide d'une table à chocs. Après 24 heures du coulage, les éprouvettes sont démoulées et conservées dans une chambre humide $(95 \% \mathrm{HR})$ à une température de $20^{\circ} \mathrm{C} \pm$ 2 jusqu'à l'âge de l'essai ou de la mise en solution.

La microstructure des mortiers a été analysée par porosimétrie au mercure après 28,90 et 360 jours de maturation en cure humide $\left(20^{\circ} \mathrm{C}\right.$ et $95 \%$ d'HR). Un prosimètre Pascal 240 a été utilisé permettant d'atteindre une pression maximale de $200 \mathrm{MPa}$ et ainsi d'accéder aux pores ayant un rayon de $3,7 \mathrm{~nm}$. Les tests sont réalisés sur des échantillons de mortiers d'environ $2 \mathrm{~cm} 3$, séchés préalablement à $50^{\circ} \mathrm{C}$ en présence de gel de silice jusqu'à masse constante ( $\Delta \mathrm{M}<0,1 \%$ en 24 heures). Ainsi la porosité totale et les courbes de distribution volumique des tailles des pores ont été mesurées.

La résistance dans des milieux agressifs est mesurée sur des éprouvettes de mortier (M0, M30 et M50). Après démoulage, les éprouvettes sont conservées en cure humide $\left(20^{\circ} \mathrm{C}\right.$ et $95 \%$ d'HR) pendant 60 jours avant d'être soumises à l'immersion dans deux types de solutions de sulfates $(5 \%$ de sulfate de sodium $(N \bar{S})$ ou
$5 \%$ de sulfate de magnésium $(M \bar{S}))$ et une solution d'eau de mer. Une série d'éprouvettes témoins a été conservée en cure humide. Notons que les solutions sont changées chaque mois. L'expansion des éprouvettes prismatique (20x20x160 mm), selon la norme ENV 196-10, a été mesurée à l'aide d'un comparateur de précision de $1 \mu \mathrm{m}$ après chaque mois, durant les 12 mois d'immersions dans les trois solutions. Par ailleurs, l'évolution de la résistance à la compression a été mesurée sur des éprouvettes $40 \times 40 \times 160 \mathrm{~mm}$, après $2,3,6$ et 10 mois d'immersion dans les sulfates de sodium et de magnésium ainsi que pour les éprouvettes témoins conservées en cure humide.

\section{Résultats et discussion}

\subsection{Microstructure}

Le tableau 2 regroupe les valeurs de la porosité volumique totale $(\mathrm{P})$, le rayon moyen de pore "Rm" et le rayon critique "Rc". Le rayon moyen est calculé en se basant sur un modèle de pore cylindrique de rayon $\mathrm{Ri}$ et de volume $\mathrm{Vi}$. $\mathrm{Si}$ « $\mathrm{S} »$ est la surface interne des pores et «V » le volume total, alors Rm est calculé par l'équation 1. Le rayon critique Rcr (ou de percolation) est défini comme le rayon correspondant au pique (ou mode de remplissage) apparent dans la courbe de distribution différentielle des volumes des pores. Ces courbes, obtenues par différentiation logarithmique, sont présentées dans la figure 1.

$$
\mathrm{R}_{\mathrm{m}}=2 \mathrm{~V} / \mathrm{S}
$$

$\mathrm{Au}$ vu des résultats, on peut voir une diminution de la porosité à l'eau avec l'âge, pour tous les mortiers étudiés. Toutefois, on peut constater une porosité plus importante, à tout âge, pour les mortiers au laitier, particulièrement, pour le M50 par rapport au mortier M0. En observant les résultats de plus prêt, on remarque que la diminution de la porosité entre 28 et 360 jours est plus importante pour le M30 et M50 que pour le M0. Cette variation est typique et est la conséquence de l'hydratation latente du laitier dans le ciment Portland. Ceci est largement confirmé dans la littérature $[6,7]$.

Comme le montre la figure 1 , à 28 jours de maturation, la distribution du volume des pores des mortiers au laitier (M30 et M50) est plus grossière que celle du mortier M0. Le rayon critique ainsi que le rayon moyen des pores sont plus élevés pour les mortiers M30 et M50 (Tableau 2). A partir de 90 jours, et particulièrement après 360 jours de maturation, la distribution de la taille des pores des mortiers au laitier a évolué considérablement vers les petits pores. En effet, les rayons moyen et critique des pores sont moins importants pour les mortiers M30 et M50 que pour le mortier M0. Ce raffinement de la porosité, plus apparent pour le mortier avec $50 \%$ de laitier est expliqué par l'hydratation du laitier qui se fait par une consommation de chaux $(\mathrm{CH})$, conduisant à la formation des "CSH" 
additionnels qui contribuent à la densification de la structure poreuse de la matrice [5].

\subsection{Résistance dans les milieux agressifs}

\subsubsection{Expansion}

Les résultats d'expansion des éprouvettes de mortier avec et sans laitier après 12 mois d'immersion dans des solutions de sulfates de sodium, de sulfates de magnésium et d'eau de mer sont présentés dans les figure 2,3 et 4 respectivement.

$\mathrm{Au}$ vu de ces résultats, on peut voir une expansion globalement plus importante des mortiers conservées dans la solution de sulfate de sodium, comparée à celle des éprouvettes conservées dans les solutions de sulfate de magnésium et d'eau de mer.

Tableau 1. Composition chimique du ciment et du laitier

\begin{tabular}{lccccccccc}
\hline & $\mathrm{CaO}$ & $\mathrm{Al}_{2} \mathrm{O}_{3}$ & $\mathrm{Fe}_{2} \mathrm{O}_{3}$ & $\mathrm{SiO}_{2}$ & $\mathrm{MgO}$ & $\mathrm{K}_{2} \mathrm{O}$ & $\mathrm{Na}_{2} \mathrm{O}$ & $\begin{array}{l}\text { Perte } \\
\text { au feu }\end{array}$ & $\mathrm{SO}_{3}$ \\
\hline Ciment & 61,30 & 5,60 & 3,80 & 18,40 & 0,90 & 0,78 & 0,42 & 2,2 & 3,30 \\
\hline Laitier & 42,84 & 9,19 & 3,44 & 41,20 & 2,12 & - & - & 0,2 & - \\
\hline
\end{tabular}
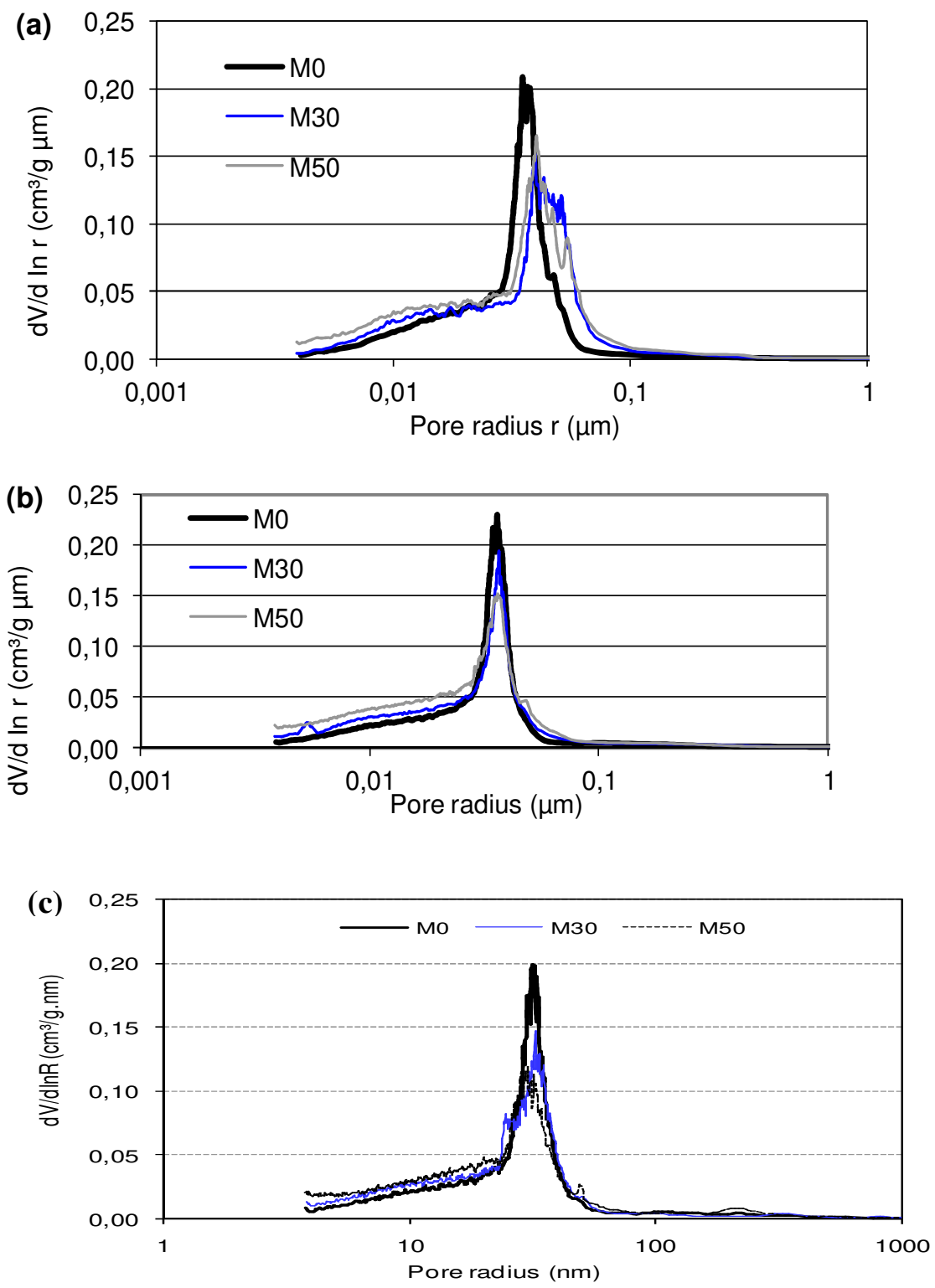

Fig. 1. Distribution des tailles des pores des mortiers au laitier après 28 jours (a), 90 jours (b) et 360 jours (c). 
Tableau 2. Porosité $(\mathrm{P})$, Rayon critique $\left(R_{c r}\right)$ et rayon moyen des pores $\left(R_{m}\right)$ des mortiers étudiés.

\begin{tabular}{cccccccccc}
\hline \multicolumn{3}{c}{28 jours } & \multicolumn{3}{c}{90 jours } & \multicolumn{3}{c}{360 jours } \\
\hline Mortiers & $\mathrm{P}(\%)$ & $R_{c r}(\mathrm{~nm})$ & $R_{m}(\mathrm{~nm})$ & $\mathrm{P} \%)$ & $R_{c r}(\mathrm{~nm})$ & $R_{m}(\mathrm{~nm})$ & $\mathrm{P}(\%)$ & $R_{c r}(\mathrm{~nm})$ & $R_{m}(\mathrm{~nm})$ \\
\hline M0 & 11.20 & 35,0 & 35,0 & 10.90 & 36,0 & 32,0 & 10.40 & 31,5 & 30,5 \\
M30 & 12.50 & 40,0 & 38,8 & 11.50 & 37,0 & 30,5 & 10.50 & 30,0 & 28,0 \\
M50 & 14.15 & 40,0 & 35,8 & 13.45 & 36,0 & 27,5 & 11.60 & 28,0 & 25,3 \\
\hline
\end{tabular}

$\mathrm{Au}$ regard des figures 2 et 3 , on constate que l'incorporation du laitier dans les mortiers, conduit à une diminution significative de l'expansion, particulièrement en solution de sulfates de sodium $(5 \% N \bar{S})$. Le mortier M0 a atteint une expansion de $0,45 \%$ après 12 mois d'immersion dans la solution $N \bar{S}$. A cette échéance, les mortiers M30 et M50 présentent respectivement, une expansion plus faible de 33 et $83 \%$ par rapport au mortier M0. Dans la solution de sulfate de magnésium $(5 \% M \bar{S})$, après 12 mois d'immersion, le M0 et le M30 ont eu une expansion similaire (de l'ordre de $0,12 \%$ par contre le mortier M50 n'a subi qu'une faible expansion de 0,02\% soit une diminution de $81 \%$ par rapport au mortier témoin. Dans la solution d'eau de mer, (figure 4), on observe une faible expansion $(0,02-0,03 \%$ après 12 mois) et peu variable entre les trois mortiers étudiés avec et sans laitier, en comparaison avec l'expansion observée dans les solutions de sulfates de sodium ou de magnésium. Toutefois, une expansion légèrement supérieure des mortiers au laitier (notamment M50) est constatée.

La variation observée reflète un comportement typique des ciments au laitier face à un environnement sulfatique. En effet, la diminution de l'expansion a été observée par plusieurs chercheurs, surtout pour des taux de laitier supérieur à 50\%, particulièrement dans un environnement de sulfates de sodium $[8,9]$.

La différence d'expansion des mortiers entre les deux solutions $(5 \% N \bar{S})$ et $(5 \% M \bar{S})$ indique bien la dissimilitude dans le mécanisme d'attaque. En effet, la détérioration du ciment par les sulfates de magnésium est contrôlée par l'attaque de magnésium - gypse sur le gel de silicate de calcium hydraté ( $\mathrm{CSH})$. La formation du gypse et de la brucite $(\mathrm{MH})$ est due aux réactions entre $M \bar{S}$ et $\mathrm{CH}, \mathrm{CSH}[10]$. Le gypse formé réagit avec l'aluminate du ciment. La brucite, formée, étant insoluble (sa solubilité est de $0,01 \mathrm{~g} / \mathrm{l}$ comparée à $1,37 \mathrm{~g} / \mathrm{l}$ pour le $\mathrm{CH})$ et ayant un bas $\mathrm{pH}$, tend à déstabiliser l'ettringite formée $[11,12]$ : ceci peut expliquer la relative faible expansion des mortiers dans la solution $\operatorname{de} M \bar{S}$. L'expansion élevée mesurée sur les mortiers immergés dans la solution de sulfate de sodium est attribuée à la formation d'ettringite secondaire.

L'expansion plus importante, observée dans les solutions de sulfate comparées à l'eau de mer, peut être attribuée à la différence du taux de diffusion des ions $\mathrm{Cl}^{-}$et $\mathrm{SO}_{4}{ }^{-2}$, celui des $\mathrm{Cl}^{-}$étant le plus élevé. Ceci permet aux ions de $\mathrm{Cl}^{-}$de pénétrer dans la matrice plus rapidement que les ions de $\mathrm{SO}_{4}{ }^{-2}$. Les chlorures réagissent ainsi avec les $\mathrm{C}_{3} \mathrm{~A}$ pour former des chloroaluminates de calcium (Sel de Friedel), diminuant ainsi la quantité de $\mathrm{C}_{3} \mathrm{~A}$ disponible pour réagir avec les ions sulfates pour former le gypse ou l'ettringite expansive [13]. BICZOK [14] a constaté que l'expansion du béton causée par des ions sulfate en eau de mer est fortement retardée par la présence de chlorures. Il faut toutefois noter que la concentration des sulfates en eau de mer (sulfates de magnésium 5,0 g/l et sulfates de calcium 1,5 g/l) est nettement moins importante que celle des solutions de $N \bar{S}$ et $M \bar{S})$ étudiées $(50 \mathrm{~g} / 1)$.

Dans un ciment au laitier, le taux de $\mathrm{C}_{3} \mathrm{~A}$ est relativement plus faible que celui d'un ciment sans ajout. Ce taux est d'autant plus faible que le pourcentage du laitier augmente. D'autre part, le laitier de part sa de la réaction pouzzolanique qui consomme de l'hydroxyde de calcium $(\mathrm{CH})$, conduit à une diminution de la formation du gypse et par conséquence, de l'ettringite. Ceci peut expliquer la diminution de l'expansion constatée dans les mortiers au laitier, même si le laitier étudié n'est que moyennement réactif. Cette amélioration peut aussi être expliquée par la microstructure plus raffinée des mortiers au laitier observée précédemment. 


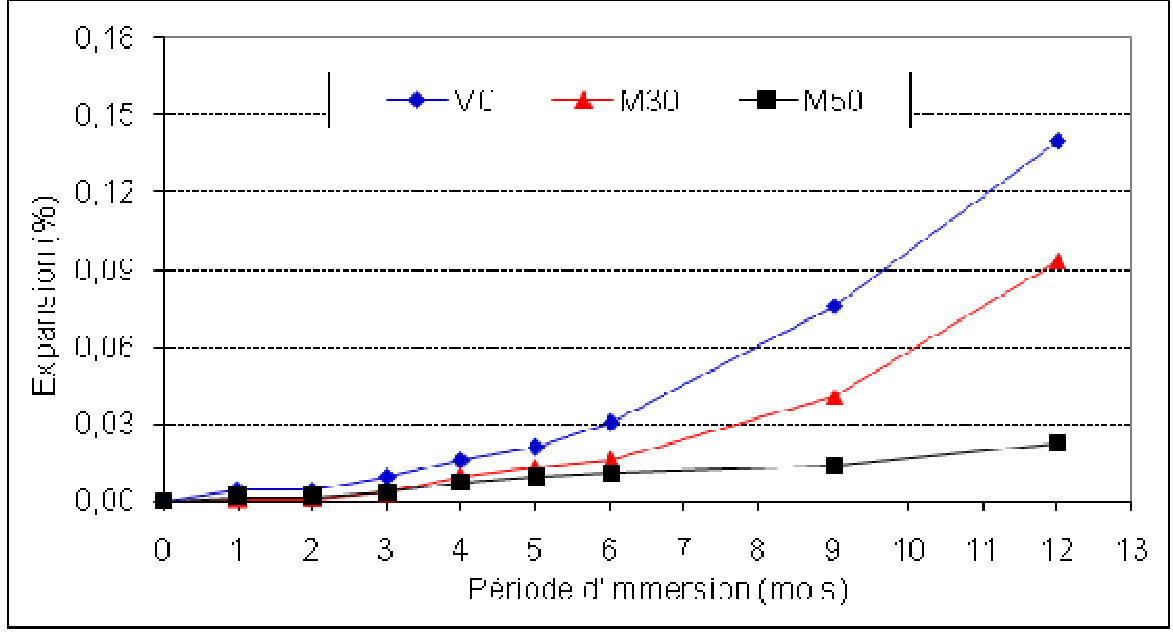

Fig. 2. Expansion des mortiers dans la solution de sulfate de sodium (5\% N $\bar{S}$ ).

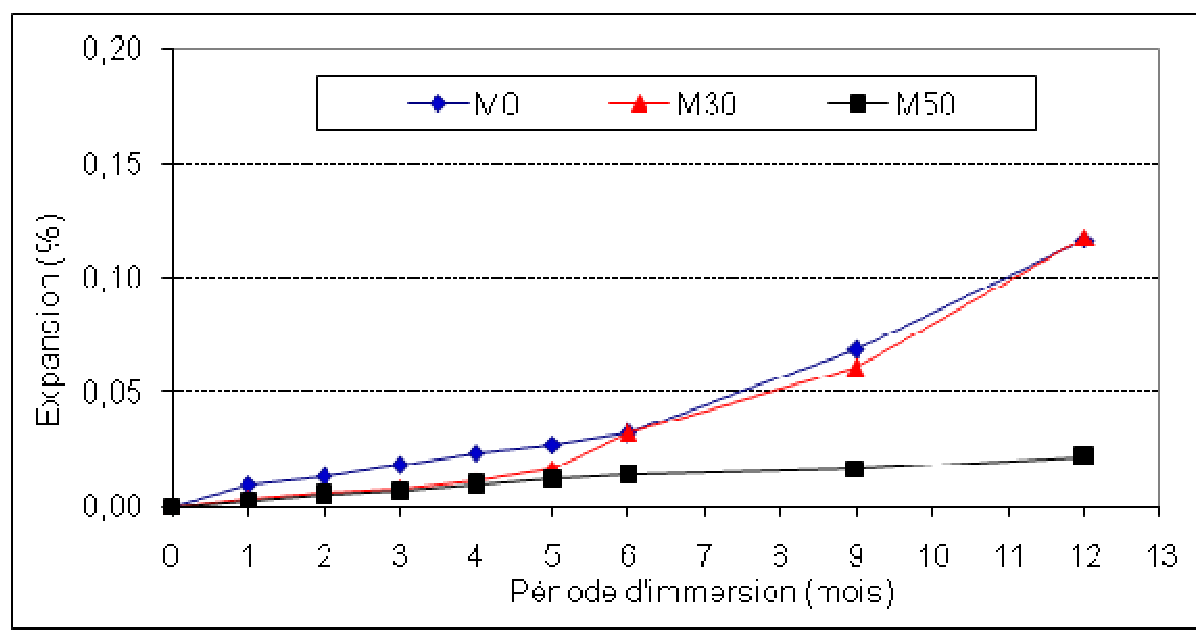

Fig. 3. Expansion des mortiers dans la solution de sulfate de magnésium (5\% $M \bar{S}$ ).

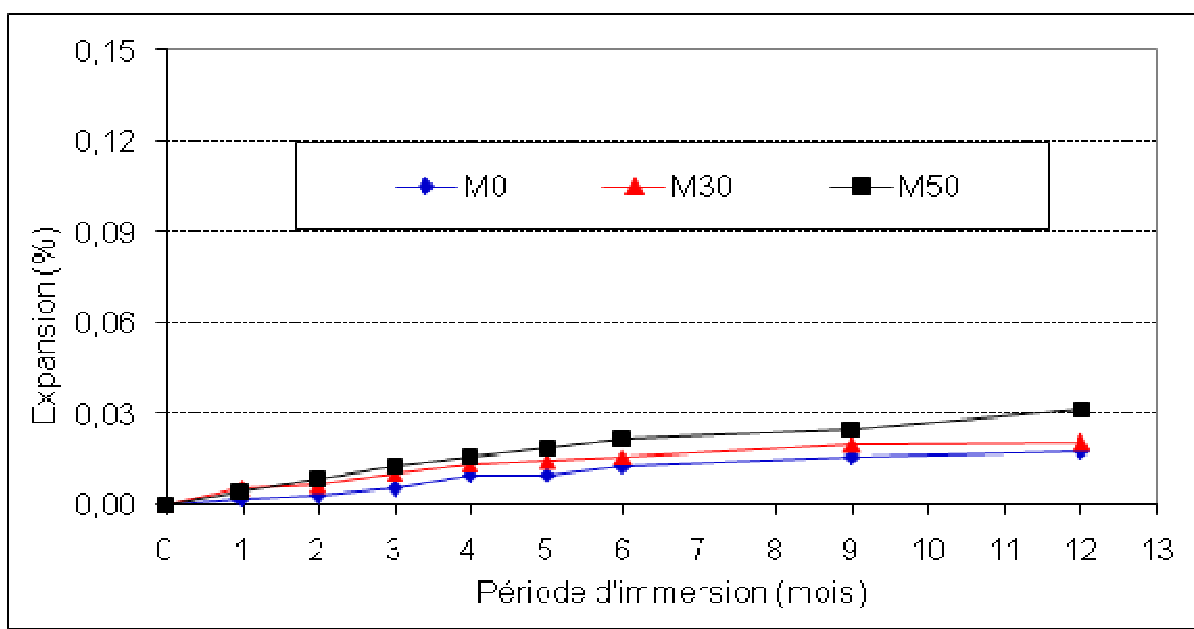

Fig. 4. Expansion des éprouvettes de mortier dans l'eau de mer 


\subsubsection{Résistance mécanique}

Le développement de la résistance en compression en fonction du temps d'immersion, dans les sulfates de sodium et de magnésium, des mortiers étudiés est représenté respectivement par les figures 5 et 6 . On peut constater une augmentation de la résistance pendant les 2 à 3 premiers mois, suivie d'une diminution, plus importante pour le mortier M0 par rapport aux mortiers M30. Cette variation est observée dans les deux solutions de sulfates, pour tous les mortiers à l'exception du M50 dont la résistance a continué son développement durant toute la période d'immersion dans les sulfates de sodium.

A fin d'estimer la perte de résistance, dans le tableau 3 on présente les rapports des résistances des éprouvettes immergées dans les sulfates par rapport à celles conservées dans $1^{\prime}$ eau à $20^{\circ} \mathrm{C}$. De ces résultats, on constate, dans la solution de sulfate de sodium, une perte de résistance à tout âge pour mortier M0. Cependant, pour les mortiers au laitier, on remarque une légère augmentation jusqu'à 90 jours d'immersion, suivie d'une perte de résistance (de 5 à $35 \%$ après 6 mois). Après 10 mois d'immersion, les mortiers M0 s'est pratiquement détérioré. A cette échéance, une perte de résistance relativement faible (18 à $25 \%$ ) est constatée pour les mortiers M30 et M50. Les mortiers conservés dans les sulfates de magnésium ont eux aussi subi une perte de résistance, notamment après 6 mois d'immersion. Par rapport aux mortiers au laitier (M30 et M50), le mortier sans ajout a accusé une perte de résistance importante de l'ordre de $73 \%$ après 10 mois d'immersion.

Par ailleurs, d'une manière générale les mortiers au laitier, ont subi des pertes de résistance plus importante dans les sulfates de magnésium que dans les sulfates de sodium. Plusieurs recherches $[15,16]$ ont montré une vulnérabilité des ciments au laitier vis-à-vis des sulfates de magnésium. Ceci s'explique par l'attaque préjudiciable des sulfates de magnésium qui se traduit, en plus de par la formation d'ettringite expansive, par la substitution partielle des CSH de la matrice par des MSH (silicates de magnésium hydratés) [17]. Les MSH étant faiblement cohésif, conduisent à une détérioration des couches surfaciques de la pâte durcie de ciment et, par conséquent, à une perte de résistance.

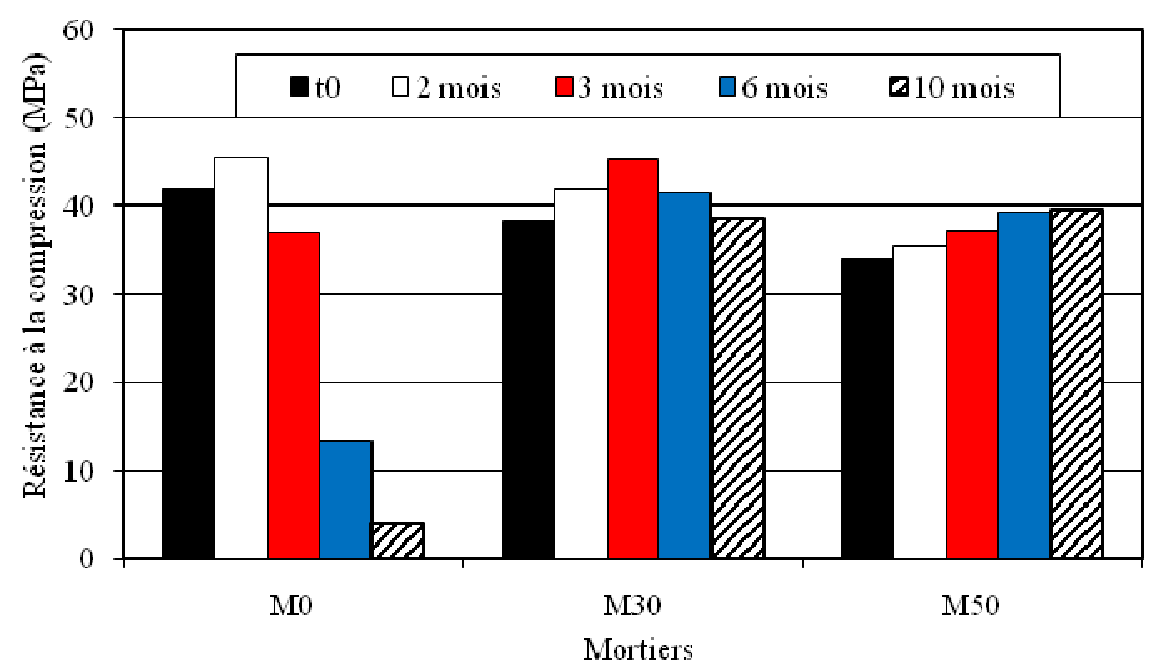

Fig. 5. Développement de la résistance à la compression des mortiers en fonction du temps d'immersion dans les sulfates de sodium $(5 \% N \bar{S})$.

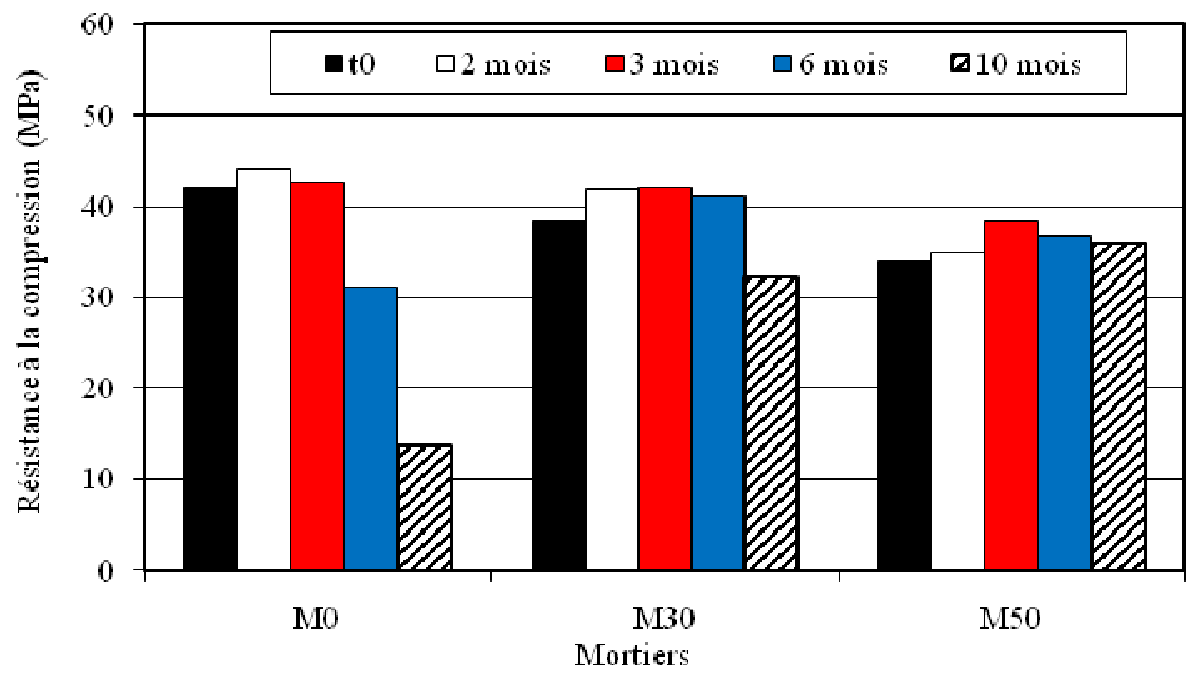

Fig. 6. Développement de la résistance à la compression des mortiers en fonction du temps d'immersion dans les sulfates de magnésium $(5 \% M \bar{S})$. 
Tableau 3 : Rapports des résistances des éprouvettes immergées dans les sulfates par rapport à celles conservées en cure humide à $20^{\circ} \mathrm{C}$.

\begin{tabular}{ccccccc}
\hline & \multicolumn{3}{c}{ Sulfates de sodium } & \multicolumn{3}{c}{ Sulfates de magnésium } \\
\hline Période d'immersion & M0 & M30 & M50 & M0 & M30 & M50 \\
\hline $\mathrm{t}_{0} *$ & 1,000 & 1,000 & 1,000 & 1,000 & 1,000 & 1,000 \\
2 & 0,974 & 1,030 & 1,026 & 0,942 & 1,025 & 1,009 \\
3 & 0,777 & 1,051 & 0,981 & 0,895 & 0,976 & 1,011 \\
6 & 0,276 & 0,963 & 0,840 & 0,639 & 0,857 & 0,848 \\
10 & 0,080 & 0,749 & 0,813 & 0,268 & 0,625 & 0,751 \\
\hline
\end{tabular}

$* \mathrm{t}_{0}$ correspond à 2 mois de conservation dans l'eau à $20^{\circ} \mathrm{C}$

\section{Conclusion}

De ce travail, les conclusions suivantes peuvent être tirées :

- La porosité des mortiers au laitier est plus importante que celle du mortier sans ajout, par contre, au long terme, les mortiers au laitier développent une distribution de la taille des pores plus raffinée, notamment pour un taux de substitution de $50 \%$.

- Même si le laitier étudié est de faible hydraulicité, les résultats obtenus prouvent que son incorporation aux ciments apporte une modification microstructurale positive conduisant à une amélioration notable de la résistance aux sulfates. Cette amélioration se traduit par une faible expansion et une bonne conservation de la résistance à la compression.

- L'effet bénéfique du laitier sur la résistance des mortiers dans les milieux agressifs est plus apparent dans les sulfates de sodium et de magnésium, que dans l'eau de mer.

\section{Références}

1. FP. Glasser, Was. Manag. J. 6, 159 (1996)

2. S. Pal, A. Mukherjee, S. Pathak, Cem. Concr. Res. 33, 1481 (2003).

3. M. Han Young, K. Hong Sam, C. Doo Sun, Constr Build Mater. 20, 725 (2006)

4. A. Bougara, C. Lynsdale, K. Ezziane, Constr Build Mater. 23, 542 (2009)

5. A. Hadj Sadok, S. Kenai, L. Courard, A. Darimont, Constr Build Mater. 25, 1018 (2011)

6. A. Cheng, R. Huang, W. Jiann-Kuo, C. ChengHsin, Mater Chem and Phys. 93, 404 (2005)

7. C. Perlot, J. Verdier, M. Carcass`es, Mater and Struct. 39, 511 (2006)

8. HT. Cao, L. Bucea, A. Ray, S. Yozghatlian, Cem. Concr. Comp. 19, 161 (1997)

9. DD. Higgins, Cem. Concr. Comp. 25, 913 (2003)

10. O.S. Alamoudi, M. Maslehuddin, M. Saadi, ACI. Mater. J. 9215 (1995)
11. Rasheeduzzafar, O.S. Al-Amoudi, SN Bduljauwad, M. Maslehuddin, ASCE. J. Mater. Civil Eng, 6, 201 (1994).

12. FM. Lea, 3rd ed, (ARNOLD, London, 1970)

13. OS. Al-Amoudi, M. Maslehuddin, Y. Abdul-Al, Constr Build Mater. 9, 25 (1995)

14. I. Biczok, Concrete Corrosion; concrete Protection (Akademiai Kiado, Budapest, 1980)

15. RS. Gollop, H. Taylor, Cem. Concr. Res. 26, 1013 (1996)

16. DD. Higgins, Cem. Concr. Comp. 25913 (2003)

17. S. Omar, B. Al-Amoudi, Cem. Concr. Comp. 24, 305 (2002) 